\title{
FUNCIONES DE LOS GRUPOS OPERATIVOS ESPAÑOLES COMO INTERMEDIARIOS DE
} LA INNOVACIÓN

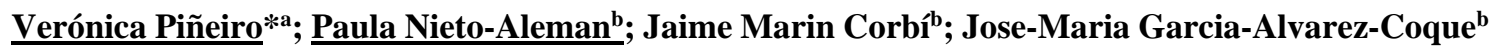 \\ a Dpto. Agronomía, Universidad Nacional del Sur (UNS), Bahía Blanca-Argentina \\ verónica.pineiro@uns.edu.ar ${ }^{\text {b }}$ Universitat Politècnica de València
}

\section{Resumen}

La política de desarrollo rural de la UE y la Asociación Europea para la Innovación sobre la Productividad y la Sostenibilidad Agrícola (EIP-AGRI) han impulsado la creación de Grupos Operativos (GO). Se promueven soluciones a problemas prácticos a través de un enfoque colaborativo del conocimiento. En este sentido, los GO pueden ser considerados como intermediarios de la innovación. Las funciones de este tipo de grupos multi-actor han sido abordadas en la literatura sobre sistemas de conocimiento agroalimentario. La presente comunicación identifica y categoriza las funciones de intermediación de los citados Grupos a partir de las opiniones de sus miembros. Una encuesta online consultó a los miembros de los GO españoles sobre las características de sus grupos y su valoración sobre el desempeño en determinadas actividades. El análisis factorial exploratorio revela que los GO actúan en tres dimensiones clave: la gestión del proceso de innovación, la articulación del conocimiento con los usuarios y las instituciones, y las propias funciones de intermediación del conocimiento. Esta caracterización puede contribuir a focalizar el tipo de resultados que se esperan de este tipo de proyectos, de cara a su evaluación.

PALABRAS CLAVE: colaboración, sistemas de innovación agrícola, intermediación del conocimiento, redes de innovación

\section{1- Introducción}

Para alcanzar los Objetivos de Desarrollo Sostenible de la Organización de las Naciones Unidas (ONU), las acciones dirigidas a la innovación tecnológica y social a través de la colaboración son fundamentales (Kanda et al., 2019; Kivimaa et al., 2019; Polzin et al., 2016; van Lente et al., 2003). Las políticas de innovación contribuyen a la puesta en práctica de modelos de innovación adaptativa a través de acciones colaborativas. Un buen ejemplo es el objeto de este estudio, los Grupos Operativos (GO) de la Asociación Europea para la Innovación en materia de Productividad y Sostenibilidad Agrícola (EIP-AGRI) ${ }^{23}$. Estas plataformas multiactores reúnen a colaboradores de diferentes ámbitos y sectores para cooperar y aportar soluciones innovadoras a los problemas agrícolas.

Leeuwis y Aarts (2011) describen la facilitación como acciones centradas en la creación de redes, el aprendizaje social y la negociación, que tienen como objetivo mejorar la sociedad. Desde esta perspectiva, a las clásicas funciones de los intermediarios, que incluyen servicios de asesoramiento, formación, documentación y gestión (Klerkx, et al., 2012), se les suma su rol como facilitadores o intermediarios de la innovación (Klerkx y Leeuwis, 2008b).

La función sistémica de los intermediarios de la innovación en lo que respecta a las políticas del sistema de innovación se ha debatido en multitud de estudios (Howells, 2006; Kilelu et al., 2011; Kilelu et al., 2013; Klerkx y Leeuwis, 2009). La mayoría identifica múltiples funciones asociadas a los intermediarios de la innovación. Estas funciones abarcan una amplia gama de formas de intermediación, desde actividades definidas y formales hasta actividades más informales y encubiertas (Kivimaa et al., 2019).

El objetivo de este estudio es contribuir al estudio de las funciones de los intermediarios de innovación mediante un análisis empírico estructurado. Para ello se analizan las percepciones de los miembros de los GO españoles sobre las funciones que han desarrollado como intermediarios de la innovación. Varios autores han avanzado en el estudio de estas funciones (Batterink et al., 2010; Howells, 2006; Jacobsson y Johnson, 2000; Kilelu et al., 2011; Klerkx y Leeuwis, 2008b; Kristjanson et al., 2009; Smits y Kuhlmann, 2004). A partir de esta base nos preguntamos cuáles de las funciones expuestas en la literatura son realmente realizadas por los $\mathrm{GO}$ españoles.

\section{Método}

\footnotetext{
${ }^{23}$ La EIP-AGRI, vinculada a las políticas de desarrollo rural dentro de los Programas de Desarrollo Rural (2014-2020), es una asociación para la innovación creada por la UE para abordar los retos sociales actuales promoviendo la interacción entre proveedores y usuarios de conocimientos para crear soluciones sostenibles y aplicables (EU SCAR, 2016).
} 
Se realizó una encuesta a los miembros de los GO autonómicos y supra-autonómicos españoles, que figuraban como dato de contacto en la base de datos de la Red Nacional Rural. La encuesta se envió por correo electrónico entre diciembre de 2018 y febrero de 2019. De las 967 encuestas enviadas, se recibieron 159 respuestas, lo que supone una tasa de respuesta del 16,4\% (error muestral de 6,5\%, nivel de confianza $90 \%, \mathrm{Z}=1.645, \mathrm{p}=\mathrm{q}=0.5)$. El objetivo de la encuesta era recoger la opinión de los miembros de los GO españoles de EIP-AGRI para conocer las funciones de facilitación que estaban llevando a cabo. Para ello se utilizaron 17 variables que expresan diferentes funciones. Las afirmaciones se evaluaron en una escala de Likert de siete puntos que iba de 1 (totalmente en desacuerdo) a 7 (totalmente de acuerdo).

Se realizó un análisis factorial exploratorio (AFE) para definir el menor número de dimensiones (variables) capaces de explicar el máximo de información contenida en los datos. Los coeficientes y los resultados de varias pruebas (determinante de la matriz, test de Bartlett, prueba de Kaiser Meyer Olkin) indican que los datos son adecuados para realizar el análisis factorial. Para este análisis se utilizó el software FACTOR (Ferrando y Lorenzo-Seva, 2014).

\section{Resultados}

Los resultados del AFE muestran que tres factores explican el 66\% de la varianza total. Estos factores están compuestos por 11 variables o acciones realizadas por estos intermediarios. A partir de estos resultados, la Tabla 1 presenta un modelo de las funciones de innovación que mejor explican el rendimiento percibido por los miembros de los GO españoles. Las variables se agrupan en tres grandes funciones que se perciben como relevantes o se consideran prioritarias para las GO españoles. Estas tres funciones son la gestión del proceso de innovación, la articulación de la demanda y, el apoyo institucional y la intermediación de la innovación. Estas funciones son altamente consistentes con varias funciones propuestas por Klerkx y Leeuwis (2009) y con la clasificación preliminar basada en la de Kilelu et al. (2011, 2013).

Tabla 1 Modelo de funciones de los intermediarios de innovación

\begin{tabular}{|c|c|}
\hline Función de innovación & Variable ítem/descripción \\
\hline $\begin{array}{l}\text { Factor 1: Gestión del } \\
\text { proceso de innovación }\end{array}$ & $\begin{array}{ll}\text { C1 } & \begin{array}{l}\text { Apoyar los proyectos y objetivos de los GO a través de nuevas } \\
\text { organizaciones }\end{array} \\
\text { I1 } & \text { Fortalecer la colaboración dentro de los GO } \\
\text { I2 } & \text { Actualizar a los actores con las acciones y avances de los GO } \\
\text { I4 } & \text { Promover mecanismos de seguimiento y evaluación }\end{array}$ \\
\hline $\begin{array}{l}\text { Factor 2: Articulación } \\
\text { de la demanda }\end{array}$ & \begin{tabular}{|ll} 
D1 & Realización de estudios prospectivos \\
D2 & Identificar las necesidades de los actores \\
D3 & Identificación de posibles soluciones
\end{tabular} \\
\hline $\begin{array}{l}\text { Factor 3: Apoyo } \\
\text { institucional e } \\
\text { intermediación en la } \\
\text { innovación }\end{array}$ & \begin{tabular}{|ll} 
S2 & Obtención de apoyo económico e institucional \\
S3 & Realización de campañas de sensibilización \\
N3 & Atracción de colaboradores externos \\
K1 & Transferencia de nuevos conocimientos y tecnologías
\end{tabular} \\
\hline
\end{tabular}

\section{Discusión y conclusiones}

El fomento de la colaboración, el intercambio de información y el desarrollo de proyectos conjuntos son actividades fundamentales para un intermediario de la innovación. El primer factor que emerge de nuestro análisis, gestión del proceso de innovación, está fuertemente relacionado con el desarrollo de capacidades (C1), entendido como las acciones que promueven nuevas organizaciones para apoyar proyectos y objetivos dentro de los GO.

El segundo factor, articulación de la demanda, combina las acciones dirigidas a la identificación de oportunidades, el desarrollo de estudios y la búsqueda de soluciones que interesen y satisfagan las necesidades de los GO y sus miembros. Estas acciones de los intermediarios de la innovación han sido citadas por varios autores (Kilelu et al., 2011, 2013; Klerkx y Leeuwis 2008c, 2009; van Lente et al., 2003; Smits y Kuhlmann 2004; Aerni, 2015).

Finalmente, el apoyo institucional e intermediación de la innovación combina variables que ayudan a crear vínculos entre los actores de la innovación. 
Una debilidad de las economías del sur de Europa, entre ellas España, es la carencia de una cultura de colaboración empresarial para las iniciativas de innovación (García-Alvarez-Coque, 2020). La política de innovación para la sostenibilidad en el marco de la EIP-AGRI apoya la colaboración para la resolución de problemas específicos. Los resultados muestran que las funciones de los GO españoles, que pueden ser consideradas como intermediarios de la innovación, pueden agruparse en tres grandes grupos. Nuestro análisis corrobora que existe un margen por recorrer en relación a funciones como la creación de capacidades y el lanzamiento de nuevos emprendimientos y proyectos de innovación orientados desde el propio sector privado.

La principal contribución de este estudio es proporcionar resultados de la comprobación empírica de la teoría de las funciones de los intermediarios de innovación. Reconocemos ciertas limitaciones del presente enfoque, que se basa principalmente en los datos autodeclarados de las opiniones de los miembros de los GO, y no en sus resultados reales. Análisis comparativos con otros países y también con carácter intertemporal podrían contextualizar mejor los resultados de los GO españoles. Estas limitaciones crean oportunidades para futuros estudios una vez que la EIP-AGRI se haya desarrollado más en España y en otros países de la UE.

Bibliografía

Aerni, P., Nichterlein, K., Rudgard, S., and Sonnino, A. (2015). "Making agricultural innovation systems (AIS) work for development in tropical countries". Sustainability, (7), 831-850. https://doi.org/10.5167/uzh-105491

Batterink, M. H., Wubben, E. F. M., Klerkx, L., and Omta, S. W. F. (2010). “Orchestrating innovation networks: The case of innovation brokers in the agri-food sector". Entrepreneurship and Regional Development, 22(1), 47-76. https://doi.org/10.1080/08985620903220512

Ferrando, Pere J., and Lorenzo-Seva, U. (2014). "El análisis factorial exploratorio de los ítems: Algunas consideraciones adicionales". Anales de Psicologia, 30(3), 1170-1175. https://doi.org/10.6018/analesps.30.3.199991

Garcia-Alvarez-Coque, J.-M., Saini, E., Esteban-Rodrigo, E., and Mas-Verdu, F. (2020). "Governance of knowledge and innovation in the Ibero-American agri-food system". Spanish Journal of Agricultural Research, 18(4), e0112. https://doi.org/10.5424/sjar/2020184-16883

Howells, J. (2006). "Intermediation and the role of intermediaries in innovation". Research Policy, 35(5), 715-728. https://doi.org/10.1016/j.respol.2006.03.005

Jacobsson, S., and Johnson, A. (2000). "The diffusion of renewable energy technology: An analytical framework and key issues for research". Energy Policy, 28(9), 625-640. https://doi.org/10.1016/S0301-4215(00)00041-0

Kanda, W., Río, P. del, Hjelm, O., and Bienkowska, D. (2019). “A technological innovation systems approach to analyse the roles of intermediaries in eco-innovation. Journal of Cleaner Production, 227, 1136-1148. https://doi.org/10.1016/j.jclepro.2019.04.230

Kilelu, C. W., Klerkx, L., and Leeuwis, C. (2013). Unravelling the role of innovation platforms in supporting co-evolution of innovation: Contributions and tensions in a smallholder dairy development programme". Agricultural Systems, $118, \quad 65-77$. https://doi.org/10.1016/j.agsy.2013.03.003

Kilelu, C. W., Klerkx, L., Leeuwis, C., and Hall, A. (2011). "Beyond knowledge brokering: an exploratory study on innovation intermediaries in an evolving smallholder agricultural system in Kenya". Knowledge Management for Development Journal, 7(1), 84-108. https://doi.org/10.1080/19474199.2011.593859

Kivimaa, P., Boon, W., Hyysalo, S., and Klerkx, L. (2019). "Towards a typology of intermediaries in sustainability transitions: A systematic review and a research agenda". Research Policy, 48(4), 1062-1075. https://doi.org/10.1016/j.respol.2018.10.006

Klerkx, Laurens, and Leeuwis, C. (2008b). "Matching demand and supply in the agricultural knowledge infrastructure: Experiences with innovation intermediaries". Food Policy, 33(3), 260-276. https://doi.org/10.1016/j.foodpol.2007.10.001

Klerkx, Laurens, and Leeuwis, C. (2009). "Establishment and embedding of innovation brokers at different innovation system levels: Insights from the Dutch agricultural sector”. Technological Forecasting and Social Change, 76(6), 849-860. https://doi.org/10.1016/j.techfore.2008.10.001 
Klerkx, Laurens, Schut, M., Leeuwis, C., and Kilelu, C. (2012). "Advances in knowledge brokering in the agricultural sector: Towards innovation system facilitation”. IDS Bulletin, 43(5), 53-60. https://doi.org/10.1111/j.1759-5436.2012.00363.x

Kristjanson, P., Reid, R. S., Dickson, N., Clark, W. C., Romney, D., Puskur, R., MacMillan, S., and Grace, D. (2009). "Linking international agricultural research knowledge with action for sustainable development." In Proceedings of the National Academy of Sciences of the United States of America (Vol. 106, Issue 13). https://doi.org/10.1073/pnas.0807414106

Leeuwis, C., \& Aarts, N. (2011). "Rethinking communication in innovation processes: creating space for change in complex systems". Journal of agricultural education and extension, 17(1), 21-36.

Polzin, F., von Flotow, P., and Klerkx, L. (2016). "Addressing barriers to eco-innovation: Exploring the finance mobilisation functions of institutional innovation intermediaries". Technological Forecasting and Social Change, 103, 34-46. https://doi.org/10.1016/j.techfore.2015.10.001

Smits, R., and Kuhlmann, S. (2004). "The rise of systemic instruments in innovation policy”. International Journal of Foresight and Innovation Policy, 1(1-2), 4-32. https://doi.org/10.1504/ijfip.2004.004621

Sutherland, L. A., Madureira, L., Dirimanova, V., Bogusz, M., Kania, J., Vinohradnik, K., Creaney, R., Duckett, D., Koehnen, T., and Knierim, A. (2017). "New knowledge networks of small-scale farmers in Europe's periphery". Land Use Policy, 63, 428-439. https://doi.org/10.1016/j.landusepol.2017.01.028

van Lente, H., Hekkert, M., Smits, R., and van Waveren, B. (2003). "Roles of Systemic Intermediaries in Transition Processes". International Journal of Innovation Management, 07(03), 247-279. https://doi.org/10.1142/s1363919603000817 